\title{
A Simplified Approach in the Study of Elliptic Differential Equations in UMD Spaces and New Applications
}

\author{
By
}

\author{
Angelo Favini*, Rabah LabBas, Stéphane Maingot, \\ Hiroki TANABE and Atsushi YaGI
}

(Università degli Studi di Bologna, Italy, Université du Havre, France, Université du Havre, France, and Osaka University, Japan)

\begin{abstract}
This paper is devoted to provide some new clarifications in the study of complete abstract second order differential equations of elliptic type given in our recent papers [8], [9] and [10]. We improve the situation in the case of the space $L^{p}(0,1 ; X)$ with a UMD Banach space $X$ by considering an approach generalizing those used in [10]. On the other hand, we give some new examples to which our theory applies.

Key Words and Phrases. Abstract differential equations of second order, Boundary value problem, UMD spaces, Bounded imaginary powers, Maximal regularity, Dore-Venni theorem.

2000 Mathematics Subject Classification Numbers. 35J25, 35J40, 47D06.
\end{abstract}

\section{Introduction and hypotheses}

Let us consider the second order abstract differential equation

$$
u^{\prime \prime}(x)+2 B u^{\prime}(x)+A u(x)=f(x), \quad x \in(0,1),
$$

with the boundary conditions

$$
\left\{\begin{array}{l}
u(0)=u_{0} \\
u(1)=u_{1}
\end{array}\right.
$$

Here $f$ is an $X$-valued function on $[0,1], X$ being a complex Banach space, $u_{0}$, $u_{1}$ are given elements of $X$ and $(A, D(A)),(B, D(B))$ are two linear operators in $X$.

This paper is devoted to provide some new clarifications in the study of (1)-(2) extending those given in our recent papers [8], [9] and [10]. We improve and clarify the situation in the case of the space $L^{p}(0,1 ; X)$ with a UMD Banach space $X$.

\footnotetext{
* The research was partially supported by italian MIUR and by University of Bologna, funds for selected research topics. It fits the program of GNAMPA.
} 
In the papers quoted above, the essential hypotheses specifying the elliptic character of equation (1) use the following one

$$
\left\{\begin{array}{l}
B^{2}-A \text { is a linear closed densely defined operator in } X, \\
\boldsymbol{R}_{-} \subset \rho\left(B^{2}-A\right) \text { and } \exists C>0: \forall \lambda \geqslant 0 \\
\left\|\left(\lambda I+B^{2}-A\right)^{-1}\right\|_{L(X)} \leqslant C /(1+\lambda),
\end{array}\right.
$$

(which is exactly the one used in Krein [17] when $B=0$ ) and some appropriate assumptions concerning the two following operators

$$
L_{0}=B-\left(B^{2}-A\right)^{1 / 2}, \quad M_{0}=-B-\left(B^{2}-A\right)^{1 / 2} .
$$

Note that $-\left(B^{2}-A\right)^{1 / 2}$ is well defined in virtue of (3).

The purpose of this paper is also to give a unified approach in the analysis of (1)-(2) by using uniquely two closed linear operators $L, M$ (not necessarily both boundedly invertible) which can be the above operators $L_{0}, M_{0}$.

We assume that $f \in L^{p}(0,1 ; X), 1<p<\infty$, and we seek for a strict solution $u$ to (1)-(2), i.e. a function $u$ such that

$$
u \in W^{2, p}(0,1 ; X) \cap L^{p}(0,1 ; D(A)), \quad \text { and } \quad u^{\prime} \in L^{p}(0,1 ; D(B)),
$$

satisfying (1) and (2).

Generally, more regularity is required for $f$ to obtain a strict solution, unless $X$ has some particular geometrical properties. This is why we assume in all this paper that

$$
X \text { is a UMD space. }
$$

We recall that a Banach space $X$ is a UMD space if and only if for some $p>1$ (and thus for all $p$ ) the Hilbert transform is continuous from $L^{p}(\boldsymbol{R} ; X)$ into itself (see Bourgain [1], Burkholder [2]). We also recall the class $\operatorname{BIP}(\alpha, X)$, see Prüss-Sohr [23]. A closed linear densely defined operator $U$ belongs to the class $\operatorname{BIP}(\alpha, X)$, where $\alpha \in[0, \pi[$ if

$$
\left\{\begin{array}{l}
]-\infty, 0[\subset \rho(U), \operatorname{ker}(U)=\{0\}, \overline{\operatorname{Im}(U)}=X \\
\text { and } \exists c \geqslant 1: \forall \lambda>0,\left\|(U+\lambda I)^{-1}\right\| \leqslant c / \lambda,
\end{array}\right.
$$

and

$$
\left\{\begin{array}{l}
\text { For all } s \in \boldsymbol{R}, U^{i s} \in L(X) \text { and } \\
\exists c \geqslant 1: \forall s \in \boldsymbol{R},\left\|U^{i s}\right\| \leqslant c e^{\alpha|s|} .
\end{array}\right.
$$

If $(P, D(P)),(Q, D(Q))$ are two linear operators, we use the notation

$$
P \subset Q,
$$

to say that $D(P) \subset D(Q)$ and $P=Q$ on $D(P)$. 
We will assume that there exist $L, M$ two closed linear operators satisfying

$$
\begin{gathered}
\left\{\begin{array}{l}
\text { i) } \quad D(L)=D(M) \\
\text { ii) } \quad D(M L)=D(L M),
\end{array}\right. \\
\begin{cases}\text { i) } & L-M \subset 2 B \\
\text { ii) } & L M=M L \subset-A,\end{cases} \\
\left\{\begin{array}{l}
\left.\exists \theta_{L}, \theta_{M} \in\right] 0, \pi / 2[\text { such that } \\
(-L) \in \operatorname{BIP}\left(\theta_{L}, X\right) ;(-M) \in \operatorname{BIP}\left(\theta_{M}, X\right),
\end{array}\right.
\end{gathered}
$$

(this implies that $\operatorname{ker}(L+M)=\{0\}$, see (12) further) and

$$
(L+M)^{-1} \text { admits a bounded extension to all } X \text {. }
$$

Our main result in this paper affirms that under the above assumptions on $L, M$, Problem (1)-(2) has a unique strict solution in $L^{p}(0,1 ; X)$ if and only if

$$
u_{0}, u_{1} \in\left(D\left(L^{2}\right), X\right)_{1 / 2 p, p}=\left(D\left(M^{2}\right), X\right)_{1 / 2 p, p} .
$$

Our techniques are based upon the Dore-Venni Theorem [6] on the sum of two closed linear operators, on the results in Prüss-Sohr paper [23] and on the reiteration Theorem in interpolation theory (see [18], [26]).

The plan of the paper is as follows.

In Section 2, we give some resulting consequences of the above assumptions. Section 3 is devoted to the main result and its proof.

Section 4 contains a comparison with the approach used in [10].

Finally in section 5 we give some examples to which our theory applies.

\section{Consequences of assumptions}

1. It is not difficult to see that assumption (7) is equivalent to

$$
\left\{\begin{array}{l}
D(L)=D(M), \\
D\left(L^{2}\right)=D\left(M^{2}\right),
\end{array}\right.
$$

and then

$$
D\left(L^{2}\right)=D\left(M^{2}\right)=D(M L)=D(M L) .
$$

2. Due to Prüss-Sohr [23] (Theorem 2, p. 437), assumption (9) imply that $L$ and $M$ generate uniformly bounded analytic semigroups in $X$

$$
\left(e^{x L}\right)_{x \geqslant 0}, \quad\left(e^{x M}\right)_{x \geqslant 0}
$$

3. On the other hand, in virtue of the celebrated Dore-Venni Theorem [6], for $C=L$ or $M$ and $g \in L^{p}(0,1 ; X)$, the application 


$$
x \mapsto C \int_{0}^{x} e^{(x-s) C} g(s) d s
$$

belongs to $L^{p}(0,1 ; X)$, if $C$ is boundedly invertible. If not, this result remains true, see Prüss-Sohr [23].

4. We deduce from the second statement of (8) that the resolvents of $L$ and $M$ commute where they exist.

5. Also, using Prüss-Sohr [23] (Theorems 4, 5, p. 441, p. 443), the commutative case of the sum theory of $L$ and $M$ applies and gives the following:

$$
-(L+M) \in \operatorname{BIP}(\theta, X) \quad \text { with } \theta=\max \left(\theta_{L}, \theta_{M}\right) .
$$

We deduce from our hypotheses (7) and (8) ii) that $L M$ is closed since we can apply Lemma 1 below. This fact and Corollary 3 of Prüss-Sohr [23], p. 444 yield

$$
L M, M L \in \operatorname{BIP}\left(\theta_{L}+\theta_{M}, X\right) .
$$

Lemma 1. Let $P$ and $Q$ be two closed operators in $X$ such that

$$
\left\{\begin{array}{l}
\rho(P) \neq \varnothing \\
D(P) \subset D(Q) \\
(P-\lambda)^{-1} Q \phi=Q(P-\lambda)^{-1} \phi \quad \text { for } \phi \in D(Q), \lambda \in \rho(P),
\end{array}\right.
$$

then $P Q$ is closed.

Proof. Let $f_{n} \in D(P Q)$ such that

$$
\left\{\begin{array}{l}
f_{n} \rightarrow f \\
(P Q) f_{n} \rightarrow g .
\end{array}\right.
$$

Then, for a given $\lambda \in \rho(P)$

$$
\begin{aligned}
Q f_{n} & =(P-\lambda)^{-1}(P-\lambda) Q f_{n} \\
& =(P-\lambda)^{-1} P Q f_{n}-\lambda(P-\lambda)^{-1} Q f_{n} \\
& =(P-\lambda)^{-1} P Q f_{n}-\lambda Q(P-\lambda)^{-1} f_{n},
\end{aligned}
$$

and, since, $Q(P-\lambda)^{-1}$ is bounded, we get

$$
Q f_{n} \rightarrow(P-\lambda)^{-1} g-\lambda Q(P-\lambda)^{-1} f=h .
$$

Hence, $f \in D(Q)$ and

$$
Q f=h .
$$


Now

$$
\begin{aligned}
h & =(P-\lambda)^{-1} g-\lambda Q(P-\lambda)^{-1} f \\
& =(P-\lambda)^{-1}(g-\lambda Q f),
\end{aligned}
$$

so $h \in D(P)$ and

$$
(P-\lambda) h=g-\lambda Q f=g-\lambda h,
$$

that is

$$
P h=g
$$

from which we deduce that $f \in D(P Q)$ and

$$
P h=P Q f=g .
$$

6. It follows then that $(L+M)$ generate a uniformly bounded analytic semigroup in $X$

$$
\left(e^{x(L+M)}\right)_{x \geqslant 0}
$$

7. It is important to remark that in virtue of Corollary 2, p. 443, in [23], one has

$$
\left\{\begin{array}{l}
L(L+M)^{-1} \text { and } M(L+M)^{-1} \text { admit } \\
\text { bounded extensions to all } X .
\end{array}\right.
$$

But there is no reason to have the same for $(L+M)^{-1}$. This is why we have assumed (10).

Note that if we suppose that $0 \in \rho(L) \cup \rho(M)$ together with (13), then (10) is fullfiled.

\section{The main abstract result}

Put $Z=e^{L+M}$, then in virtue of Lunardi [19] (p. 60), $I-Z$ has a bounded inverse. Thus, the complete analysis of Problem (1)-(2) is equivalent to the one of

$$
\left\{\begin{array}{l}
v^{\prime \prime}(x)+2 B v^{\prime}(x)+A v(x)=(I-Z) f(x), \quad x \in(0,1), \\
v(0)=(I-Z) u_{0} \\
v(1)=(I-Z) u_{1}
\end{array}\right.
$$

by setting $v=(I-Z) u$. Now, taking into account the representation formula used in [10], we set 


$$
\begin{aligned}
v(x)= & e^{x M} u_{0}+(L+M)^{-1} \int_{0}^{x} e^{(x-s) M}(I-Z) f(s) d s \\
& -(L+M)^{-1} e^{x M} \int_{0}^{1} e^{s L} f(s) d s-e^{(1-x) L} e^{M} f_{0} \\
& +e^{(1-x) L} u_{1}+(L+M)^{-1} \int_{x}^{1} e^{(s-x) L}(I-Z) f(s) d s \\
& -(L+M)^{-1} e^{(1-x) L} \int_{0}^{1} e^{(1-s) M} f(s) d s-e^{x M} e^{L} f_{1},
\end{aligned}
$$

where

$$
f_{0}=u_{0}-(L+M)^{-1} \int_{0}^{1} e^{s L} f(s) d s, \quad f_{1}=u_{1}-(L+M)^{-1} \int_{0}^{1} e^{(1-s) M} f(s) d s .
$$

Note that in the representation of $v$, we find some symmetry in the exchange

$$
\left(x, u_{0}, f, M\right) \leftrightarrow\left(1-x, u_{1}, f(1-\cdot), L\right) .
$$

This is why we write

$$
\begin{aligned}
v(x)= & S\left(x, u_{0}, f, M\right)-R\left(1-x, f_{0}, L\right) \\
& +S\left(1-x, u_{1}, f(1-\cdot), L\right)-R\left(x, f_{1}, M\right)
\end{aligned}
$$

where, for $x \in(0,1), \phi \in X, g \in L^{p}(0,1 ; X)$ and $C=L$ or $M$

$$
\begin{aligned}
S(x, \phi, g, C)= & e^{x C} \phi+(L+M)^{-1} \int_{0}^{x} e^{(x-s) C}(I-Z) g(s) d s \\
& -(L+M)^{-1} e^{x C} \int_{0}^{1} e^{s(L+M-C)} g(s) d s
\end{aligned}
$$

and

$$
R(x, \phi, C)=e^{x C} e^{L+M-C} \phi .
$$

Lemma 2. Assume (4), (7) (10). Let $C=L$ or $M$ and $\phi$ a given element in $X$. Then for the regular term $R(\cdot, \phi, C)$ we have

$$
A R(\cdot, \phi, C), L^{2} R(\cdot, \phi, C), M^{2} R(\cdot, \phi, C) \in L^{p}(0,1 ; X) .
$$

Proof. For $C=L$ or $M$, one has

$$
e^{L+M-C} \phi \in D\left(L^{2}\right)=D\left(M^{2}\right)=D(M L)=D(M L),
$$

and for a.e. $x \in(0,1)$ 


$$
A R(x, \phi, C)=-M L R(x, \phi, C)=-e^{x C} M L e^{L+M-C} \phi,
$$

so $A R(\cdot, \phi, C)$ is bounded and thus in $L^{p}(0,1 ; X)$. It is the same for $L^{2} R(\cdot, \phi, C)$ and $M^{2} R(\cdot, \phi, C)$.

Lemma 3. Assume (4) and (7) (10). Then, for $C=L$ or $M$ and $\lambda_{0} \in \rho(C)$

$$
x \mapsto\left(C-\lambda_{0} I\right) C e^{x C} \phi \in L^{p}(0,1 ; X)
$$

if and only if

$$
\phi \in\left(D\left(C^{2}\right), X\right)_{1 / 2 p, p} .
$$

In this case, there exists $c>0$ such that

$$
\left\|\left(C-\lambda_{0} I\right) C e^{\cdot C} \phi\right\|_{L^{p}(X)} \leqslant c\|\phi\|_{\left(D\left(C^{2}\right), X\right)_{1 / 2 p, p}} .
$$

Proof. It is well known that

$$
\begin{aligned}
x \mapsto C^{2} e^{x C} \phi \in L^{p}(0,1 ; X) & \Leftrightarrow x \mapsto x^{1 / p} C^{2} e^{x C} \phi \in L_{*}^{p}(0,1 ; X) \\
& \Leftrightarrow \phi \in\left(D\left(C^{2}\right), X\right)_{1 / 2 p, p},
\end{aligned}
$$

since $C$ generates an analytic semigroup (see Triebel [26], p. 96). Now, let $\lambda_{0} \in \rho(C)$. If

$$
\phi \in\left(D\left(C^{2}\right), X\right)_{1 / 2 p, p}
$$

then

$$
\begin{aligned}
\left\|C e^{x C} \phi\right\|_{X} & =\left\|C e^{C} \phi+\int_{1}^{x} C^{2} e^{s C} \phi d s\right\|_{X} \\
& \leqslant c\|\phi\|_{X}+\int_{x}^{1}\left\|C^{2} e^{s C} \phi\right\|_{X} d s,
\end{aligned}
$$

and, by Hölder inequality, we obtain

$$
\begin{aligned}
\left\|C e^{\cdot C} \phi\right\|_{X} & \leqslant c\|\phi\|_{X}+(1-x)^{1-1 / p}\left\|C^{2} e^{\cdot C} \phi\right\|_{L^{p}(0,1 ; X)} \\
& \leqslant c\|\phi\|_{\left(D\left(C^{2}\right), X\right)_{1 / 2 p, p}}
\end{aligned}
$$

from which one has

$$
\left(C-\lambda_{0} I\right) C e^{\cdot C} \phi=C^{2} e^{\cdot C} \phi-\lambda_{0} C e^{\cdot C} \phi \in L^{p}(0,1 ; X),
$$

and the desired estimate.

Conversely if $\left(C-\lambda_{0} I\right) C e^{\cdot C} \phi \in L^{p}(0,1 ; X)$ then

$$
C e^{\cdot C} \phi=\left(C-\lambda_{0} I\right)^{-1}\left(C-\lambda_{0} I\right) C e^{\cdot C} \phi \in L^{p}(0,1 ; X),
$$


and

$$
C^{2} e^{\cdot C} \phi=\left(C-\lambda_{0} I\right) C e^{\cdot C} \phi+\lambda_{0} C e^{\cdot C} \phi \in L^{p}(0,1 ; X),
$$

so

$$
\phi \in\left(D\left(C^{2}\right), X\right)_{1 / 2 p, p} .
$$

Lemma 4. Assume (4), (7) (10). Let $C=L$ or $M, g \in L^{p}(0,1 ; X)$ and $\phi$ a given element in $X$. Then for the term $S(\cdot, \phi, g, C)$ we have

1. $A S(\cdot, \phi, g, C) \in L^{p}(0,1 ; X)$ if and only if

$$
\phi \in\left(D\left(L^{2}\right), X\right)_{1 / 2 p, p}=\left(D\left(M^{2}\right), X\right)_{1 / 2 p, p} .
$$

2. $M^{2} S(\cdot, \phi, g, C) \in L^{p}(0,1 ; X)$ if and only if

$$
\phi \in\left(D\left(L^{2}\right), X\right)_{1 / 2 p, p}=\left(D\left(M^{2}\right), X\right)_{1 / 2 p, p} .
$$

3. $L^{2} S(\cdot, \phi, g, C) \in L^{p}(0,1 ; X)$ if and only if

$$
\phi \in\left(D\left(L^{2}\right), X\right)_{1 / 2 p, p}=\left(D\left(M^{2}\right), X\right)_{1 / 2 p, p} .
$$

Moreover when $\phi \in\left(D\left(C^{2}\right), X\right)_{1 / 2 p, p}$ there exists $c>0$ such that

$$
\begin{aligned}
& \|A S(\cdot, \phi, g, C)\|_{L^{p}(X)}+\left\|M^{2} S(\cdot, \phi, g, C)\right\|_{L^{p}(X)}+\left\|L^{2} S(\cdot, \phi, g, C)\right\|_{L^{p}(X)} \\
& \quad \leqslant c\left(\|g\|_{L^{p}(X)}+\|\phi\|_{\left(D\left(C^{2}\right), X\right)_{1 / 2 p, p}}\right) .
\end{aligned}
$$

Proof. Statement 1. We set, for a.e. $x \in(0,1)$

$$
\begin{aligned}
\mathscr{L}(x, g, C) & =L M(L+M)^{-1} \int_{0}^{x} e^{(x-s) C}(I-Z) g(s) d s \\
& =(L+M-C)(L+M)^{-1} C \int_{0}^{x} e^{(x-s) C}(I-Z) g(s) d s .
\end{aligned}
$$

Since

$$
(L+M-C)(L+M)^{-1} \in L(X),
$$

one has

$$
\mathscr{L}(\cdot, g, C) \in L^{p}(0,1 ; X)
$$

Consider also

$$
\mathscr{M}(x, g, C)=L M(L+M)^{-1} e^{x C} \int_{0}^{1} e^{s(L+M-C)} g(s) d s,
$$

then 


$$
\mathscr{M}(\cdot, g, C) \in L^{p}(0,1 ; X)
$$

since

$$
\begin{aligned}
\mathscr{M}(x, g, C)= & L M(L+M)^{-1} e^{x C} \int_{0}^{x} e^{s(L+M-C)} g(s) d s \\
& +L M(L+M)^{-1} e^{x C} \int_{0}^{1-x} e^{(1-s)(L+M-C)} g(1-s) d s \\
= & \mathscr{L}\left(x, e^{\cdot C} e^{\cdot(L+M-C)}(I-Z)^{-1} g, C\right) \\
& +e^{x C} e^{x(L+M-C)} \mathscr{L}\left(1-x,(I-Z)^{-1} g(1-\cdot), L+M-C\right) .
\end{aligned}
$$

To conclude, choose $\lambda_{0} \in \rho(C)$ and write

$$
\begin{aligned}
A S(x, \phi, g, C)= & -L M e^{x C} \phi-L M(L+M)^{-1} \int_{0}^{x} e^{(x-s) C}(I-Z) g(s) d s \\
& +L M(L+M)^{-1} e^{x C} \int_{0}^{1} e^{s(L+M-C)} g(s) d s \\
= & -(L+M-C)\left(C-\lambda_{0} I\right)^{-1}\left(C-\lambda_{0} I\right) C e^{x C} \phi \\
& -\mathscr{L}(x, g, C)+\mathscr{M}(x, g, C),
\end{aligned}
$$

then by Lemma 3, we obtain statement 1 .

Statements 2 and 3 are obtained by the same way. Now, in virtue of Lemma 3 and from the Dore-Venni Theorem one obtains the quoted estimate.

Theorem 5. Assume (4) and (7) (10). Let $f \in L^{p}(0,1 ; X)$ with $1<p<\infty$. One has

1. If there exists a function $u$ such that

$$
u \in W^{2, p}(0,1 ; X) \cap L^{p}(0,1 ; D(L M)), \quad u^{\prime} \in L^{p}(0,1 ; D(L-M)),
$$

and satisfying (1)-(2) then $u$ is unique.

In this case $u$ is called the $(L, M)$-strict solution of Problem (1)-(2).

2. Problem (1)-(2) has a $(L, M)$-strict solution $u$ if and only if

$$
u_{0}, u_{1} \in\left(D\left(M^{2}\right), X\right)_{1 / 2 p, p}=\left(D\left(L^{2}\right), X\right)_{1 / 2 p, p} .
$$

In this case, there exists $c>0$ such that

$$
\begin{aligned}
& \left\|u^{\prime \prime}\right\|_{L^{p}(X)}+\left\|(L-M) u^{\prime}\right\|_{L^{p}(X)}+\|L M u\|_{L^{p}(X)} \\
& \quad \leqslant c\left(\|f\|_{L^{p}(X)}+\left\|u_{0}\right\|_{\left(D\left(M^{2}\right), X\right)_{1 / 2 p, p}}+\left\|u_{1}\right\|_{\left(D\left(M^{2}\right), X\right)_{1 / 2 p, p}}\right) .
\end{aligned}
$$


Note that $u$ is a strict solution of Problem (1)-(2) in the classical sense since $u$ satisfies (1)-(2) with

$$
u \in W^{2, p}(0,1 ; X) \cap L^{p}(0,1 ; D(A)), \quad u^{\prime} \in L^{p}(0,1 ; D(B)) .
$$

Proof. 1. It is enough to prove the result when $f=0, u_{0}=0$ and $u_{1}=0$.

We set

$$
\left\{\begin{array}{l}
v=L(L+M)^{-1} u+(L+M)^{-1} u^{\prime} \\
w=M(L+M)^{-1} u-(L+M)^{-1} u^{\prime} .
\end{array}\right.
$$

Then

$$
\begin{aligned}
v^{\prime} & =L(L+M)^{-1} u^{\prime}+(L+M)^{-1} u^{\prime \prime} \\
& =L(L+M)^{-1} u^{\prime}-(L+M)^{-1}\left(2 B u^{\prime}+A u\right) \\
& =L(L+M)^{-1}-(L-M)(L+M)^{-1} u^{\prime}+M L(L+M)^{-1} u \\
& =M(L+M)^{-1} u^{\prime}+M L(L+M)^{-1} u \\
& =M v
\end{aligned}
$$

and

$$
\begin{aligned}
w^{\prime} & =M(L+M)^{-1} u^{\prime}-(L+M)^{-1} u^{\prime \prime} \\
& =M(L+M)^{-1} u^{\prime}+(L+M)^{-1}\left(2 B u^{\prime}+A u\right) \\
& =M(L+M)^{-1}+(L-M)(L+M)^{-1} u^{\prime}-L M(L+M)^{-1} u \\
& =L(L+M)^{-1} u^{\prime}-L M(L+M)^{-1} u \\
& =-L w .
\end{aligned}
$$

So if we set $\tilde{w}=w(1-\cdot)$ then

$$
v^{\prime}=M v, \quad \tilde{w}^{\prime}=L \tilde{w},
$$

but $L$ and $M$ generate analytic semigroups thus

$$
v=e^{x M} \xi_{0}, \quad \tilde{w}^{\prime}=e^{x L} \xi_{1} .
$$

Finally

$$
\begin{aligned}
u & =v+w \\
& =v+\tilde{w}(1-\cdot) \\
& =e^{x M} \xi_{0}+e^{(1-x) L} \xi_{1},
\end{aligned}
$$


and from $u_{0}=0$ and $u_{1}=0$ we get

$$
\left\{\begin{array}{l}
0=\xi_{0}+e^{L} \xi_{1} \\
0=e^{M} \xi_{0}+\xi_{1}
\end{array}\right.
$$

so $\xi_{0}=\xi_{1}=0$ and $u=0$.

2. Recall that we set $u=(I-Z)^{-1} v$ where

$$
\begin{aligned}
v(x)= & S\left(x, u_{0}, f, M\right)-R\left(1-x, f_{0}, L\right) \\
& +S\left(1-x, u_{1}, f(1-\cdot), L\right)-R\left(x, f_{1}, M\right) .
\end{aligned}
$$

From Lemma 2, one has

$$
A R\left(1-\cdot, f_{0}, L\right), A R\left(\cdot, f_{1}, M\right) \in L^{p}(0,1 ; X),
$$

and Lemma 4 gives

$$
\left\{\begin{array}{l}
A S\left(\cdot, u_{0}, f, M\right) \in L^{p}(0,1 ; X) \Leftrightarrow u_{0} \in\left(D\left(M^{2}\right), X\right)_{1 / 2 p, p} \\
A S\left(1-\cdot, u_{1}, f(1-\cdot), L\right) \in L^{p}(0,1 ; X) \Leftrightarrow u_{1} \in\left(D\left(L^{2}\right), X\right)_{1 / 2 p, p} .
\end{array}\right.
$$

Summarizing, we obtain

$$
A v \in L^{p}(0,1 ; X) \Leftrightarrow u_{0}, u_{1} \in\left(D\left(M^{2}\right), X\right)_{1 / 2 p, p}=\left(D\left(L^{2}\right), X\right)_{1 / 2 p, p} .
$$

On the other hand

$$
\begin{aligned}
2 B v^{\prime}(x)= & 2 B M S\left(x, u_{0}, f, M\right)+2 B L R\left(1-x, f_{0}, L\right) \\
& -2 B L S\left(1-x, u_{1}, f(1-\cdot), L\right)-2 B M R\left(x, f_{1}, M\right) \\
= & (L-M) M S\left(x, u_{0}, f, M\right)+(L-M) L R\left(1-x, f_{0}, L\right) \\
& -(L-M) L S\left(1-x, u_{1}, f(1-\cdot), L\right)-(L-M) M R\left(x, f_{1}, M\right),
\end{aligned}
$$

or equivalently

$$
\begin{aligned}
2 B v^{\prime}(x)= & -\left(A+M^{2}\right) S\left(x, u_{0}, f, M\right)+\left(L^{2}+A\right) R\left(1-x, f_{0}, L\right) \\
& -\left(L^{2}+A\right) S\left(1-x, u_{1}, f(1-\cdot), L\right)+\left(A+M^{2}\right) R\left(x, f_{1}, M\right) .
\end{aligned}
$$

And for $u_{0}, u_{1} \in\left(D\left(M^{2}\right), X\right)_{1 / 2 p, p}=\left(D\left(L^{2}\right), X\right)_{1 / 2 p, p}$ one has

$$
2 B v^{\prime}(\cdot) \in L^{p}(0,1 ; X) .
$$

But $L$ and $M$ generate analytic semigroups and from (15)

$$
A v=-L M v, \quad 2 B v^{\prime}=(L-M) v .
$$

So $v$ and thus $u$ have the desired regularities. We conclude, by showing that $v$ satisfies (14). One has 


$$
\begin{aligned}
v^{\prime \prime}(x)= & M^{2} S\left(x, u_{0}, f, M\right)-L^{2} R\left(1-x, f_{0}, L\right) \\
& +L^{2} S\left(1-x, u_{1}, f(1-\cdot), L\right)-M^{2} R\left(x, f_{1}, M\right) \\
& +(L+M)(L+M)^{-1} f(x),
\end{aligned}
$$

and

$$
\begin{aligned}
A v(x)= & A S\left(x, u_{0}, f, M\right)-A R\left(1-x, f_{0}, L\right) \\
& +A S\left(1-x, u_{1}, f(1-\cdot), L\right)-A R\left(x, f_{1}, M\right),
\end{aligned}
$$

then

$$
\begin{aligned}
v^{\prime \prime}(x)+ & 2 B v^{\prime}(x)+A v(x) \\
= & {\left[M^{2}+2 B M+A\right] S\left(x, u_{0}, f, M\right)-\left[L^{2}-2 B L+A\right] R\left(1-x, f_{0}, L\right) } \\
& +\left[L^{2}-2 B L+A\right] S\left(1-x, u_{1}, f, L\right)-\left[M^{2}+2 B M+A\right] R\left(x, f_{1}, M\right) \\
& +f(x) \\
= & f(x) .
\end{aligned}
$$

\section{Moreover}

$$
\begin{aligned}
v(0) & =S\left(0, u_{0}, f, M\right)-R\left(1, f_{0}, L\right)+S\left(1, u_{1}, f(1-\cdot), L\right)-R\left(0, f_{1}, M\right) \\
& =(I-Z) u_{0}
\end{aligned}
$$

and

$$
\begin{aligned}
v(1) & =S\left(1, u_{0}, f, M\right)-R\left(0, f_{0}, L\right)+S\left(0, u_{1}, f(1-\cdot), L\right)-R\left(1, f_{1}, M\right) \\
& =(I-Z) u_{1} .
\end{aligned}
$$

Remark 1. The present approach does not allow to treat the elliptic boundary value problem in the square $\Omega=] 0,1[\times] 0,1[$

$$
\begin{aligned}
& \frac{\partial^{2} u}{\partial x^{2}}+2 b \frac{\partial^{2} u}{\partial x \partial y}+a \frac{\partial^{2} u}{\partial y^{2}}=f(x, y), \quad(x, y) \in \Omega, \\
& \left.u\right|_{\Gamma_{i}}=g_{i}, \quad i=1,2,3,4
\end{aligned}
$$

where

$$
\begin{array}{ll}
\Gamma_{1}=\{(0, y) ; 0<y<1\}, & \Gamma_{2}=\{(x, 0) ; 0<x<1\}, \\
\Gamma_{3}=\{(1, y) ; 0<y<1\}, & \Gamma_{4}=\{(x, 1) ; 0<x<1\},
\end{array}
$$


so that $\partial \Omega=\bigcup_{i=1}^{4} \bar{\Gamma}_{i}, f \in L^{p}(\Omega), 1<p<\infty, g_{i}$ are suitable functions on $\bar{\Gamma}_{i}$ and the real coefficients satisfy the ellipticity condition $a-b^{2}>0$. Notice that the domain is in fact non smooth.

Introduce the new variables

$$
\xi=y-b x, \quad \eta=-\left(a-b^{2}\right)^{1 / 2} x
$$

and suppose $b>0$. Then equation (16) reads

$$
\frac{\partial^{2} v}{\partial \xi^{2}}+\frac{\partial^{2} v}{\partial \eta^{2}}=g(\xi, \eta), \quad(\xi, \eta) \in \Sigma
$$

where

$$
\begin{aligned}
& v(\xi, \eta)=u\left(-\frac{1}{\sqrt{a-b^{2}}} \eta, \xi-\frac{b}{\sqrt{a-b^{2}}} \eta\right), \\
& g(\xi, \eta)=\frac{1}{a-b^{2}} f\left(-\frac{\eta}{\sqrt{a-b^{2}}}, \xi-\frac{b}{\sqrt{a-b^{2}}} \eta\right)
\end{aligned}
$$

and $\Sigma$ is the parallerogram whose vertices are $(0,0),\left(-b,-\sqrt{a-b^{2}}\right)$, $\left(1-b,-\sqrt{a-b^{2}}\right),(1,0)$.

Using P. Grisvard [15], Theorem 4.4.3.7 and Corollary 4.4.3.8, p. 233, it is possible to show a result as follows.

Proposition 6. Let $b>0, a-b^{2}>0,1<p<\infty, 1 / p+1 / q=1$. If

$$
\frac{2}{q \pi}\left(\pi-\arctan \frac{\sqrt{a-b^{2}}}{b}\right) \leqslant 1
$$

then for all $f \in L^{p}(\Omega), g_{i} \in W_{p}^{2-1 / p}(0,1), g_{1}(1)=g_{4}(0), g_{2}(0)=g_{1}(0), g_{2}(1)=$ $g_{3}(0), g_{3}(1)=g_{4}(1)$, problem (16), (17) has a unique solution $u \in W_{p}^{2}(\Omega)$.

\section{Comparison with the approach in [10]}

In this section we will illustrate our above abstract theory by building a typical model of a pair of operators $(L, M)$ satisfying assumptions (7) (10).

Let us assume that operators $A, B$ are such that

(AB1) $\left\{\begin{array}{l}B^{2}-A \text { is closed densely defined with } \boldsymbol{R}_{-} \subset \rho\left(B^{2}-A\right) \text { and } \\ \exists c>0 \forall \lambda \geqslant 0,\left\|\left(\lambda I+B^{2}-A\right)^{-1}\right\|_{L(X)} \leqslant c /(1+\lambda),\end{array}\right.$

(then it is well known that $-\left(B^{2}-A\right)^{1 / 2}$ is the infinitesimal generator of an analytic semigroup)

$$
\left\{\begin{array}{l}
D\left(\left(B^{2}-A\right)^{1 / 2}\right) \subseteq D(B) \text { and } \forall y \in D(B) \\
B\left(B^{2}-A\right)^{-1 / 2} y=\left(B^{2}-A\right)^{-1 / 2} B y
\end{array}\right.
$$


and

(AB3)

$$
\left\{\begin{array}{l}
\left.\exists \theta_{L_{0}}, \theta_{M_{0}} \in\right] 0, \pi / 2[: \\
\left(-L_{0}\right) \in \operatorname{BIP}\left(\theta_{L_{0}}, X\right) ;\left(-M_{0}\right) \in \operatorname{BIP}\left(\theta_{M_{0}}, X\right)
\end{array}\right.
$$

where

$$
\left\{\begin{array}{l}
M_{0}=-B-\left(B^{2}-A\right)^{1 / 2} \\
L_{0}=B-\left(B^{2}-A\right)^{1 / 2}
\end{array}\right.
$$

Lemma 7. Suppose that (AB1), (AB2) and (AB3) hold. Then, one has

$$
\left\{\begin{array}{l}
D\left(M_{0}\right)=D\left(L_{0}\right)=\left(B^{2}-A\right)^{1 / 2} \\
D\left(M_{0} L_{0}\right)=D\left(L_{0} M_{0}\right)=D\left(B^{2}-A\right) \\
M_{0} L_{0}=L_{0} M_{0} \subset-A,
\end{array}\right.
$$

and

$$
\left(L_{0}+M_{0}\right)^{-1}=-\frac{1}{2}\left(B^{2}-A\right)^{-1 / 2} \in L(X) .
$$

Proof. We only show $D\left(M_{0} L_{0}\right) \subset D\left(B^{2}-A\right)$, since the other parts are easily seen. Let $u \in D\left(M_{0} L_{0}\right)$. Then

$$
u \in D\left(L_{0}\right)=D\left(\left(B^{2}-A\right)^{1 / 2}\right), \quad L_{0} u \in D\left(M_{0}\right)=D\left(\left(B^{2}-A\right)^{1 / 2}\right) .
$$

Let

$$
v=\left(B^{2}-A\right)^{1 / 2}\left(I-L_{0}\right) u .
$$

Noting that $1 \in \rho\left(L_{0}\right)$ in view of $(\mathrm{AB} 3)$ set

$$
w=\left(B^{2}-A\right)^{-1 / 2}\left(I-L_{0}\right)^{-1} v .
$$

Then

$$
\left(B^{2}-A\right)^{1 / 2} w=\left(I-L_{0}\right)^{-1} v \in D\left(L_{0}\right)=D\left(\left(B^{2}-A\right)^{1 / 2}\right) .
$$

Hence

$$
w \in D\left(B^{2}-A\right) .
$$

One has $\left(B^{2}-A\right)^{1 / 2} w \in D(B)$ by virtue of (19) and (AB2). Again using (AB2) one deduces that

$$
B w=B\left(B^{2}-A\right)^{-1 / 2}\left(B^{2}-A\right)^{1 / 2} w=\left(B^{2}-A\right)^{-1 / 2} B\left(B^{2}-A\right)^{1 / 2} w .
$$

Hence 


$$
B w \in D\left(\left(B^{2}-A\right)^{1 / 2}\right) \quad \text { and } \quad\left(B^{2}-A\right)^{1 / 2} B w=B\left(B^{2}-A\right)^{1 / 2} w .
$$

Therefore

$$
\begin{aligned}
v & =\left(I-L_{0}\right)\left(B^{2}-A\right)^{1 / 2} w=\left(I-B+\left(B^{2}-A\right)^{1 / 2}\right)\left(B^{2}-A\right)^{1 / 2} w \\
& =(I-B)\left(B^{2}-A\right)^{1 / 2} w+\left(B^{2}-A\right) w \\
& =\left(B^{2}-A\right)^{1 / 2}(I-B) w+\left(B^{2}-A\right) w \\
& =\left(B^{2}-A\right)^{1 / 2}\left[I-B+\left(B^{2}-A\right)^{1 / 2}\right] w=\left(B^{2}-A\right)^{1 / 2}\left(I-L_{0}\right) w .
\end{aligned}
$$

It follows from (18) and (20) that

$$
u=w \in D\left(B^{2}-A\right) .
$$

Hence, under (4), (AB1), (AB2) and (AB3), our Theorem 5 applies.

Note that, we do not need $D(A) \subset D\left(B^{2}\right)$ and the bounded invertibility of $A, L_{0}$ and $M_{0}$ as in [10].

In particular, in examples given in [10], we also can consider concrete elliptic operators $A$ not boundedly invertible as those defined by boundary Neumann conditions or even when $A=0$.

\section{Applications}

Example 1. In this example, we describe a general model to which our previous theory applies. Given a linear operator $C$ (not necessarily boundedly invertible) such that

$$
-C \in \operatorname{BIP}(\alpha, X) \quad \text { with } \alpha \in] 0, \pi / 2[,
$$

and consider $A, B$ defined by

$$
\left\{\begin{array}{l}
A=2 C-3 C^{2} \\
B=C-I .
\end{array}\right.
$$

Now, by taking $L=3 C-2 I$ and $M=C$, we verify that all the assumptions are fulfilled on $L$ and $M$ and so we can apply our previous results as well.

This example allows us to consider the cases of concrete applications where $A$ could be not invertible. In fact, one has

$$
A=C(2 I-3 C)=(2 I-3 C) C
$$

from which we deduce that the invertibility of $A$ implies the one of $C$. 
Example 2. Let $-A$ be a positive definite self-adjoint operator in a Hilbert space $X$, and $B=\rho(-A)^{1 / 2}, \rho$ a real number. Then, it is easy to show that the operators $L$ and $M$ defined by

$$
L=\left(\rho-\left(\rho^{2}+1\right)^{1 / 2}\right)(-A)^{1 / 2}, \quad M=\left(-\rho-\left(\rho^{2}+1\right)^{1 / 2}\right)(-A)^{1 / 2}
$$

satisfy assumptions $(7) \sim(10)$.

Example 3. Let $A$ and $B$ be differential operators with constant coefficients such that

$$
\begin{aligned}
& A=-\sum_{|\alpha|=4} a_{\alpha}\left(\frac{\partial}{\partial x}\right)^{\alpha}, \quad \sum_{|\alpha|=4} a_{\alpha} \xi^{\alpha}=\sum_{|\alpha|=4} \bar{a}_{\alpha} \xi^{\alpha}>0 \quad \forall \xi \neq 0, \\
& B=-\sum_{i, j=1}^{n} b_{i j} \frac{\partial^{2}}{\partial x_{i} \partial x_{j}}+1, \quad\left\{b_{i j}\right\} \text { is a positive definite symmetric matrix. }
\end{aligned}
$$

Consider these operators in $L^{p}\left(\boldsymbol{R}^{n}\right), 1<p<\infty$. Hence $D(A)=W^{4, p}\left(\boldsymbol{R}^{n}\right)$, $D(B)=W^{2, p}\left(\boldsymbol{R}^{n}\right)$. We are going to show that this pair of operators $A$ and $B$ satisfies the assumptions (AB1), (AB2) and (AB3) of section 4. Note that $0 \notin \rho(A)$, and hence the result of the previous paper [10] cannot be applied.

It is known that $(\mathrm{AB} 1)$ holds. Let

$$
A(\xi)=-\sum_{|\alpha|=4} a_{\alpha} \xi^{\alpha}, \quad B(\xi)=\sum_{i, j=1}^{n} b_{i j} \xi_{i} \xi_{j}+1 .
$$

Then $(\mathscr{F}(A u))(\xi)=A(\xi) \hat{u}(\xi)$ and $(\mathscr{F}(B u))(\xi)=B(\xi) \hat{u}(\xi)$ for $u \in D(A)$ and $u \in D(B)$ respectively, where $\mathscr{F}$ denotes the Fourier tansform and $\hat{u}=\mathscr{F} u$. Clearly

$$
D\left(B^{2}-A\right)=W^{4, p}\left(\boldsymbol{R}^{n}\right), \quad D\left(\left(B^{2}-A\right)^{1 / 2}\right)=W^{2, p}\left(\boldsymbol{R}^{n}\right)=D(B) .
$$

Since $A$ and $B$ are operators with constant coefficients, it is easy to show that (AB2) holds with the aid of the Fourier tansform or by using the Dunford integral expression of $\left(B^{2}-A\right)^{-1 / 2}$. Set

$$
H(\xi)=-B(\xi)+\left(B(\xi)^{2}-A(\xi)\right)^{1 / 2} .
$$

Evidently $H(\cdot)$ is a $C^{\infty}$ function such that $H(\xi)>0$ for $\xi \neq 0$ and $H(0)=0$. Let the operator $H$ be defined by

$$
\begin{aligned}
& D(H)=\left\{u \in L^{p}\left(\boldsymbol{R}^{n}\right) ; \mathscr{F}^{-1}(H(\cdot) \hat{u}) \in L^{p}\left(\boldsymbol{R}^{n}\right)\right\}, \\
& H u=\mathscr{F}^{-1}(H(\cdot) \hat{u}) .
\end{aligned}
$$


Lemma 8. As $\xi \rightarrow 0$

$$
\left(\frac{\partial}{\partial \xi}\right)^{\alpha} H(\xi)= \begin{cases}O\left(|\xi|^{4-|\alpha|}\right) & 0 \leqslant|\alpha| \leqslant 4 \\ O(1) & |\alpha| \geqslant 5 \text { and }|\alpha| \text { is even } \\ O(|\xi|) & |\alpha| \geqslant 5 \text { and }|\alpha| \text { is odd }\end{cases}
$$

$$
H(\xi)^{-1}=O\left(|\xi|^{-4}\right) .
$$

Proof. Since

$$
H(\xi)=B(\xi)\left\{\left(1-\frac{A(\xi)}{B(\xi)^{2}}\right)^{1 / 2}-1\right\} \sim-\frac{A(\xi)}{2 B(\xi)} \quad \text { as } \xi \rightarrow 0
$$

one observes that (21) for $|\alpha|=0$ and (22) hold. It follows from

$$
\frac{\partial H(\xi)}{\partial \xi_{j}}=\left\{B(\xi)\left(B(\xi)^{2}-A(\xi)\right)^{-1 / 2}-1\right\} \frac{\partial B(\xi)}{\partial \xi_{j}}-\frac{1}{2}\left(B(\xi)^{2}-A(\xi)\right)^{-1 / 2} \frac{\partial A(\xi)}{\partial \xi_{j}},
$$

and

$$
\begin{aligned}
& B(\xi)\left(B(\xi)^{2}-A(\xi)\right)^{-1 / 2}-1=\left(1-\frac{A(\xi)}{B(\xi)^{2}}\right)^{-1 / 2}-1 \\
& \sim \frac{A(\xi)}{2 B(\xi)^{2}}=O\left(|\xi|^{4}\right) \quad \text { as } \xi \rightarrow 0
\end{aligned}
$$

that

$$
\frac{\partial H(\xi)}{\partial \xi_{j}}=O\left(|\xi|^{3}\right) \quad \text { as } \xi \rightarrow 0,
$$

which is $(21)$ for $|\alpha|=1$. As is easily seen

(24) $\left(\frac{\partial}{\partial \xi}\right)^{\alpha}\left(B(\xi)^{2}-A(\xi)\right)= \begin{cases}O(1) & 0 \leqslant|\alpha| \leqslant 4 \text { and }|\alpha| \text { is even } \\ O(|\xi|) & 0 \leqslant|\alpha| \leqslant 4 \text { and }|\alpha| \text { is odd as } \xi \rightarrow 0 . \\ 0 & |\alpha| \geqslant 5\end{cases}$

This and (23) imply

$$
\begin{aligned}
\frac{\partial^{2} H(\xi)}{\partial \xi_{j} \partial \xi_{k}}= & \left\{\frac{\partial B(\xi)}{\partial \xi_{k}}\left(B(\xi)^{2}-A(\xi)\right)^{-1 / 2}\right. \\
& \left.-\frac{1}{2} B(\xi)\left(B(\xi)^{2}-A(\xi)\right)^{-3 / 2} \frac{\partial}{\partial \xi_{k}}\left(B(\xi)^{2}-A(\xi)\right)\right\} \frac{\partial B(\xi)}{\partial \xi_{j}} \\
& +\left\{B(\xi)\left(B(\xi)^{2}-A(\xi)\right)^{-1 / 2}-1\right\} \frac{\partial^{2} B(\xi)}{\partial \xi_{j} \partial \xi_{k}}
\end{aligned}
$$




$$
\begin{aligned}
& +\frac{1}{4}\left(B(\xi)^{2}-A(\xi)\right)^{-3 / 2} \frac{\partial}{\partial \xi_{k}}\left(B(\xi)^{2}-A(\xi)\right) \frac{\partial A(\xi)}{\partial \xi_{j}} \\
& -\frac{1}{2}\left(B(\xi)^{2}-A(\xi)\right)^{-1 / 2} \frac{\partial^{2} A(\xi)}{\partial \xi_{j} \partial \xi_{k}}=O\left(|\xi|^{2}\right) \quad \text { as } \xi \rightarrow 0 .
\end{aligned}
$$

This is $(21)$ for $|\alpha|=2$. Set

$$
G(\xi)=\left(B(\xi)^{2}-A(\xi)\right)^{1 / 2}
$$

Then, for $|\alpha| \geqslant 3$

$$
(\partial / \partial \xi)^{\alpha} H(\xi)=(\partial / \partial \xi)^{\alpha} G(\xi) .
$$

Denoting by $D$ any of the differentiations $\partial / \partial \xi_{j}, j=1, \ldots, n$, and expressing the derivatives of $G^{2}=B^{2}-A$ symbolically one observes with the aid of (24)

$$
\begin{aligned}
& 2 G \cdot D G=D\left(B^{2}-A\right)=O(|\xi|) \Rightarrow D G=O(|\xi|), \\
& 2(D G)^{2}+2 G \cdot D^{2} G=D^{2}\left(B^{2}-A\right)=O(1) \Rightarrow D^{2} G=O(1), \\
& 6 D G \cdot D^{2} G+2 G \cdot D^{3} G=D^{3}\left(B^{2}-A\right)=O(|\xi|) \Rightarrow D^{3} G=O(|\xi|), \\
& 6\left(D^{2} G\right)^{2}+8 D G \cdot D^{3} G+2 G \cdot D^{4} G=D^{4}\left(B^{2}-A\right)=\mathrm{const} \\
& \Rightarrow D^{4} G=O(1) .
\end{aligned}
$$

Continuing this process we obtain that

$$
\left(\frac{\partial}{\partial \xi}\right)^{\alpha} G(\xi)=\left\{\begin{array}{ll}
O(1) & |\alpha| \text { is even } \\
O(|\xi|) & |\alpha| \text { is odd }
\end{array} \text { as } \xi \rightarrow 0 .\right.
$$

It follows from (25) and (26) that (21) for $|\alpha| \geqslant 3$ holds. The proof is complete.

Lemma 9. One has for any multi-index $\alpha$

$$
(\partial / \partial \xi)^{\alpha} H(\xi)=O\left(|\xi|^{2-|\alpha|}\right) \quad \text { as }|\xi| \rightarrow \infty .
$$

Proof. This is easily verified noting that $B(\cdot)$ is a polynomial of second order and using again the symbolical expression of the derivatives of $G^{2}$.

Proposition 10.

$$
D(H)=W^{2, p}\left(\boldsymbol{R}^{n}\right) .
$$

Proof. Clearly there exists a positive constant $c_{0}$ such that

$$
\sum_{|\alpha|=4} a_{\alpha} \xi^{\alpha} \geqslant c_{0}\left(\sum_{i, j=1}^{n} b_{i j} \xi_{i} \xi_{j}\right)^{2} .
$$


Hence

$$
\begin{aligned}
1+H(\xi) & =-\sum_{i, j=1}^{n} b_{i j} \xi_{i} \xi_{j}+\left[\left(\sum_{i, j=1}^{n} b_{i j} \xi_{i} \xi_{j}+1\right)^{2}+\sum_{|\alpha|=4} a_{\alpha} \xi^{\alpha}\right]^{1 / 2} \\
& >-\sum_{i, j=1}^{n} b_{i j} \xi_{i} \xi_{j}+\left[\left(\sum_{i, j=1}^{n} b_{i j} \xi_{i} \xi_{j}\right)^{2}+\sum_{|\alpha|=4} a_{\alpha} \xi^{\alpha}\right]^{1 / 2} \\
& \geqslant-\sum_{i, j=1}^{n} b_{i j} \xi_{i} \xi_{j}+\sqrt{1+c_{0}} \sum_{i, j=1}^{n} b_{i j} \xi_{i} \xi_{j} \\
& =\left(\sqrt{1+c_{0}}-1\right) \sum_{i, j=1}^{n} b_{i j} \xi_{i} \xi_{j} \geqslant c_{1}|\xi|^{2}
\end{aligned}
$$

with some positive constant $c_{1}$. Therefore, in view of Lemma 9 it can be seen without difficulty that

$$
\sup _{\xi \in \boldsymbol{R}^{n}}|\xi|^{|\alpha|}\left|\left(\frac{\partial}{\partial \xi}\right)^{\alpha} \frac{1+|\xi|^{2}}{1+H(\xi)}\right|<\infty \quad \forall \alpha .
$$

Hence with the aid of the Fourier multiplier theorem by Mikhlin [20] one can easily show that $D(H) \subset W^{2, p}\left(\boldsymbol{R}^{n}\right)$. The opposite inclusion relation is shown analogously.

Corollary 11.

$$
H=-B+\left(B^{2}-A\right)^{1 / 2}
$$

\section{Proposition 12.}

$$
H \in \operatorname{BIP}\left(L^{p}\left(\boldsymbol{R}^{n}\right), \theta\right) \quad \forall \theta>0 .
$$

Proof. Suppose that $H u=0$. Then $H(\cdot) \hat{u}=0$. Since $H(\xi)>0$ for $\xi \neq 0, \hat{u}$ is a distribution whose support consists only of the origin at the most. Hence $u$ is a polynomial belonging to $L^{p}\left(\boldsymbol{R}^{n}\right)$. This implies $u=0$. Thus we have proved $N(H)=\{0\}$. Next suppose that $v \in L^{p^{\prime}}\left(\boldsymbol{R}^{n}\right), \quad p^{\prime}=$ $p /(p-1)$, and $(H u, v)=0 \forall u \in W^{2, p}\left(\boldsymbol{R}^{n}\right)$. Then for any rapidly decreasing function $u$ one has

$$
0=(H u, v)=(H(\cdot) \hat{u}, \hat{v})=(\hat{u}, H(\cdot) \hat{v}) .
$$

Hence $H(\cdot) \hat{v}=0$. This implies $v=0$ as above. Thus it has been shown that Im $H$ is dense in $L^{p}\left(\boldsymbol{R}^{n}\right)$.

In the next step we show that $(-\infty, 0) \subset \rho(H)$ and 


$$
\begin{aligned}
& \left\|(t+H)^{-1}\right\| \leqslant C / t \quad \forall t>0, \\
& \left\|H^{i s}\right\| \leqslant C_{\theta} e^{\theta|s|} \quad \forall s \in \boldsymbol{R},
\end{aligned}
$$

where $\theta$ is an arbitrary positive number. For that purpose we show that the following inequalities hold for any multi-indices $\alpha$

$$
\begin{aligned}
& \sup _{\xi \in \boldsymbol{R}^{n}}|\xi|^{|\alpha|}\left|\left(\frac{\partial}{\partial \xi}\right)^{\alpha} \frac{1}{t+H(\xi)}\right| \leqslant \frac{C_{\alpha}}{t} \\
& \sup _{\xi \in \boldsymbol{R}^{n}}|\xi|^{\alpha}\left|\left(\frac{\partial}{\partial \xi}\right)^{\alpha} H(\xi)^{i s}\right| \leqslant C_{\alpha} \sum_{k=0}^{|\alpha|-1}|i s(i s-1)(i s-2) \cdots(i s-k)| .
\end{aligned}
$$

Then in view of the Fourier multiplyer theorem by Mikhlin [20] one obtains the desired inequalities (27) and (28). Note that in view of (29) for any $f \in L^{p}\left(\boldsymbol{R}^{n}\right), \quad u=\mathscr{F}^{-1}\left[(t+H(\cdot))^{-1} \hat{f}\right]$ is the unique solution of the equation $(t+H) u=f$.

Proof of (29). First we consider the case where $|\xi|$ is small. By virtue of Lemma 8 one observes

$$
\left|\frac{\partial}{\partial \xi_{j}} \frac{1}{t+H(\xi)}\right|=\left|-\frac{\partial H(\xi)}{\partial \xi_{j}} \frac{1}{(t+H(\xi))^{2}}\right| \leqslant \frac{C|\xi|^{3}}{\left(t+|\xi|^{4}\right)^{2}}<\frac{C}{t} \frac{1}{|\xi|} .
$$

In order to estimates the higher order derivatives of $(t+H(\xi))^{-1}$ one expresses them symbolically as

$$
\begin{aligned}
D(t+H)^{-1}= & -D H \cdot(t+H)^{-2}, \\
D^{2}(t+H)^{-1}= & -D^{2} H \cdot(t+H)^{-2}+2(D H)^{2} \cdot(t+H)^{-3}, \\
D^{3}(t+H)^{-1}= & -D^{3} H \cdot(t+H)^{-2}+6 D H \cdot D^{2} H \cdot(t+H)^{-3} \\
& -6(D H)^{3} \cdot(t+H)^{-4},
\end{aligned}
$$

Then it can be shown without difficulty that the following inequality holds.

$$
\sup _{|\xi| \leqslant 1}|\xi|^{|\alpha|}\left|\frac{1}{t+H(\xi)}\right| \leqslant \frac{C_{\alpha}}{t} \text {. }
$$

The terms with $D^{\alpha} H,|\alpha| \geqslant 5$ as a factor, e.g. $-D^{5} H \cdot(t+H)^{-2}$ in $D^{5}(t+H)^{-1}$, have better estimates than necessary, since $D^{\alpha} H(\xi)=O(1)$ or $O(|\xi|)$ according as $|\alpha|$ is even or odd in view of Lemma 8 instead of $D^{\alpha} H(\xi)=O\left(|\xi|^{4-|\alpha|}\right)$.

The following inequalities are analogously established with the aid of Lemma 9 and (31). 


$$
\sup _{|\xi| \geqslant 1}|\xi|^{|\alpha|}\left|\left(\frac{\partial}{\partial \xi}\right)^{\alpha} \frac{1}{t+H(\xi)}\right| \leqslant \frac{C_{\alpha}}{t} .
$$

The inequality (29) follows from (32) and (33).

Proof of (30). We begin with the case where $|\xi|$ is small. By virtue of Lemma 8 one gets

$$
\left|\frac{\partial H(\xi)^{i s}}{\partial \xi_{j}}\right|=\left|i s H(\xi)^{i s-1} \frac{\partial H(\xi)}{\partial \xi_{j}}\right|=|s| H(\xi)^{-1}\left|\frac{\partial H(\xi)}{\partial \xi_{j}}\right| \leqslant C|s||\xi|^{-1} .
$$

Hence

$$
\sup _{|\xi| \leqslant 1}|\xi|\left|\frac{\partial H(\xi)^{i s}}{\partial \xi_{j}}\right| \leqslant C|s|
$$

Again symbolically

$$
\begin{aligned}
D H^{i s}= & i s H^{i s-1} \cdot D H, \\
D^{2} H^{i s}= & i s(i s-1) H^{i s-2} \cdot(D H)^{2}+i s H^{i s-1} \cdot D^{2} H, \\
D^{3} H^{i s}= & i s(i s-1)(i s-2) H^{i s-3} \cdot(D H)^{3} \\
& +3 i s(i s-1) H^{i s-2} \cdot D H \cdot D^{2} H+i s H^{i s-1} \cdot D^{3} H,
\end{aligned}
$$

With the aid of (34) one obtains without difficulty

$$
\sup _{|\xi| \leqslant 1}|\xi|^{\alpha}\left|\left(\frac{\partial}{\partial \xi}\right)^{\alpha} H(\xi)^{i s}\right| \leqslant C_{\alpha} \sum_{k=0}^{|\alpha|-1}|i s(i s-1)(i s-2) \ldots(i s-k)| .
$$

Again the terms with a factor $D^{\alpha} H(\xi),|\alpha| \geqslant 5$, have better estimates than necessary.

Next we consider the case where $|\xi|$ is large. Analogously using the symbolical expression (34) of the derivatives of $H(\xi)$ is one easily deduces

$$
\sup _{|\xi| \geqslant 1}|\xi|^{|\alpha|}\left|\left(\frac{\partial}{\partial \xi}\right)^{\alpha} H(\xi)^{i s}\right| \leqslant C_{\alpha} \sum_{k=0}^{|\alpha|-1}|i s(i s-1)(i s-2) \ldots(i s-k)| .
$$

The inequalies (35) and (36) yield (30).

It is easier to show that analogous results hold for $B+\left(B^{2}-A\right)^{1 / 2}$, since $B(\xi)+\left(B(\xi)^{2}-A(\xi)\right)^{1 / 2}$ does not vanish.

Remark 2. If $p=2$, we can use Parseval's equality instead of the Fourier multiplier theorem. In this case $-B+\left(B^{2}-A\right)^{1 / 2}$ is nonnegative selfadjoint 
and $B+\left(B^{2}-A\right)^{1 / 2}$ is positive definite selfadjoint, and $\left( \pm B+\left(B^{2}-A\right)^{i s}\right)^{1 / 2}$ is unitary.

\section{References}

[1] Bourgain, J., Some remarks on Banach spaces in which martingale difference sequences are unconditional, Ark. Mat., 21 (1983), 163-168.

[2] Burkholder, D. L., A geometrical characterisation of Banach spaces in which martingale difference sequences are unconditional, Ann. Probab., 9 (1981), 997-1011.

[3] Campiti, M., Metafune, G. and Pallara, D., Degenerate Self-adjoint Evolution Equations on the Unit Interval, Semigroup Forum, 57 (1998), 1-36.

[4] Da Prato, G., Abstract Differential Equations, Maximal Regularity and Linearization, Proc. Sympos. Pure Math., 45, Amer. Math. Soc., Providence, RI, 1986, 359-370.

[ 5 ] Da Prato, G. and Grisvard, P., Sommes d'Opérateurs Linéaires et Equations Différentielles Opérationnelles, J. Math. Pures Appl. IX Ser., 54 (1975), 305-387.

[ 6 ] Dore, G. and Venni, A., On the Closedness of the Sum of two Closed Operators, Math. Z., 196 (1987), 189-201.

[7] El Haial, A. and Labbas, R., On the Ellipticity and Solvability of Abstract Second-order Differential Equation, Electron. J. Differential Equations, 2001 (2001), No. 57, 1-18.

[ 8 ] Favini, A., Labbas, R., Tanabe, H. and Yagi, A., On the Solvability of Complete Abstract Differential Equations of Elliptic Type, Funkcial. Ekvac., 47 (2004), 205-224.

[9] Favini, A., Labbas, R., Maingot, S., Tanabe, H. and Yagi, A., On the Solvability and Maximal Regularity of Complete Abstract Differential Equations of Elliptic Type, Funkcial. Ekvac., 47 (2004), 423-452.

[10] Favini, A., Labbas, R., Maingot, S., Tanabe, H. and Yagi, A., Complete abstract differential equations of elliptic type in UMD spaces, Funkcial. Ekvac., 49 (2006), 193-214.

[11] Favini, A. and Romanelli, S., Analytic Semigroups on $C([0,1])$ Generated by Some Classes of Second Order Differential Operators, Semigroup Forum, 56 (1998), 367-372.

[12] Favini, A. and Triggiani, R., Analytic and Gevrey Class Semigroups Generated by $-A+i B$, and Applications, in "Differential Equations in Banach Spaces", Proceeding of the Bologna conferences, G. Dore, A. Favini, E. Obrecht, A. Venni, eds., M. Dekker, New York, 1993, 93-114.

[13] Goldstein, J. A., Semigroups of Linear Operators and Applications, Oxford University Press, Oxford, New York, 1985.

[14] Grisvard, P., Spazi di Tracce e Applicazioni, Rend. Mat. (6), 5 (1972), 657-729.

[15] Grisvard, P., Elliptic problems in non smooth domains, Pitman, Boston, 1985.

[16] Kato, T., Perturbation Theory for Linear Operators, Springer-Verlag, Berlin, Heidelberg, New York, 1966.

[17] Krein, S. G., Linear Differential Equations in Banach Space, Moscow, 1967; English Translation: AMS, Providence, 1971.

[18] Lions, J. L. and Peetre, J., Sur une classe d'espaces d'interpolation, Inst. Hautes Études Sci. Publ. Math., 19 (1964), 5-68.

[19] Lunardi, A., Analytic Semigroups and Optimal Regularity in Parabolic Problems, Birkhäuser, Basel, 1995.

[20] Mikhlin, S. G., On the multipliers of Fourier integrals, Doklady Akad. Nauk SSSR (N.S.), 109 (1956), 701-703.

[21] Miklavčič, M., Applied Functional Analysis and Partial Differential Equations, World Scientific, Singapore, 1998. 
[22] Pazy, A., Semigroups of Linear Operators and Applications to Partial Differential Equations, Springer-Verlag, Berlin, Heidelberg, Tokyo, 1983.

[23] Prüss, J. and Sohr, H., On Operators with Bounded Imaginary Powers in Banach Spaces, Math. Z., 203 (1990), 429-452.

[24] Seeley, R., Norms and Domains of the Complex Powers $\left(A_{B}\right)^{z}$, Amer. J. Math., 93 (1971), 299-309.

[25] Tanabe, H., Equations of Evolution, Pitman, London, San Francisco, Melbourne, 1979.

[26] Triebel, H., Interpolation Theory, Function Spaces, Differential Operators, Amsterdam, North Holland, 1978.

[27] Yosida, K., Functional Analysis, Springer, 1965.

nuna adreso:

Angelo Favini

Dipartimento di Matematica

Università degli Studi di Bologna

Piazza di Porta S. Donato, 5, 40126 Bologna

Italy

E-mail: favini@dm.unibo.it

Rabah Labbas

Laboratoire de Mathématiques

Faculté des Sciences et Techniques

Université du Havre

B.P 540, 76058 Le Havre Cedex

France

E-mail: rabah.labbas@univ-lehavre.fr

Stéphane Maingot

Laboratoire de Mathématiques

Faculté des Sciences et Techniques

Université du Havre

B.P 540, 76058 Le Havre Cedex

France

E-mail: stephane.maingot@univ-lehavre.fr

Hiroki Tanabe

Hirai Sanso 12-13

Takarazuka, 665-0817

Japan

E-mail: h7tanabe@jttk.zaq.ne.jp

Atsushi Yagi

Department of Applied Physics

Osaka University

Osaka 565-0871

Japan

E-mail: yagi@ap.eng.osaka-u.ac.jp

(Ricevita la 9-an de novembro, 2006) 